\title{
Posición Natural de Cabeza y su relación con el Plano de Frankfurt en cefalometría ortodóncica.
}

\section{Natural head position and its relationship with the Frankfurt Plane in orthodontic cephalometry.}

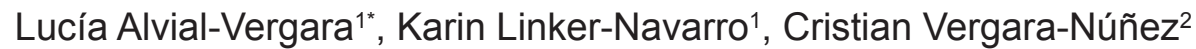

\author{
1.Práctica Privada, Santiago, Chile. \\ 2. Departamento del Niño y Ortopedia \\ Dentomaxilar, Facultad de Odontología, \\ Universidad de Chile, Santiago, Chile.
}

* Correspondencia Autor: Lucía Alvial Vergara Dirección: San Ignacio de Loyola 425, dpto. 612, Santiago, Chile | Dirección: Particular: San Ignacio de Loyola 425, dpto. 612 | Código Postal: 8330528 | E-mail: lucia.alvial@ug.uchile.cl

Trabajo recibido el 01/08/2020

Trabajo revisado 27/11/2020

Aprobado para su publicación el 02/01/2021

\section{RESUMEN}

Objetivo: el objetivo de este estudio fue determinar la discrepancia del Plano de Frankfurt evaluado en fotogrametría y en cefalometría de radiografías laterales de cráneo respecto a la horizontal verdadera. Materiales y métodos: el estudio consideró a 34 voluntarios de la clínica del Postítulo de Ortodoncia y Ortopedia Dento Máxilo Facial de la Facultad de Odontología de la Universidad de Chile. Se utilizaron sus radiografías laterales de cráneo orientadas según el Plano de Frankfurt paralelo al piso y se les tomó una fotografía facial de perfil estandarizada en Posición Natural de Cabeza. Se trazó el Plano de Frankfurt y la Horizontal Verdadera en ambos exámenes y se midió el ángulo formado entre estos planos. Resultados: al comparar los ángulos mediante T-test, no hubo diferencia estadística. Conclusiones: mediante la aplicación de un protocolo sistematizado tanto para el registro fotográfico como para la obtención de la Posición Natural de Cabeza, se puede lograr que el Plano de Frankfurt sea prácticamente coincidente en radiografías laterales de cráneo y en fotografías clínicas, demostrando su uso hasta el día de hoy en la ortodoncia moderna.

PALABRAS CLAVE

Plano de Frankfurt; Posición natural de cabeza; Horizontal verdadera; Cefalometría; Fotogrametría.

Int. J. Inter. Dent Vol. 14(3); 218-221, 2021.

\begin{abstract}
Objective: the aim of this study was to determine the Frankfurt plane variation evaluated in photogrammetry and lateral skull x-ray cephalometry in relation to the true horizontal. Materials and methods: the study included 34 volunteers from the Universidad de Chile Dento-maxillofacial Orthodontics and Orthopedics Postgraduate Clinic. Their lateral skull radiographs, oriented according to the Frankfurt Plane parallel to the floor, were used and a standardized profile facial photograph was taken in Natural Head Position. The Frankfurt Plane and True Horizontal were drawn in both examinations and the angle formed between these planes was measured. Results: when comparing the angles through T-test, there was no statistical difference. Conclusions: through the application of a systematized protocol for both photographic record and natural head position obtained, the Frankfurt Plane can be practically coincident in lateral skull x-rays and clinical photographs, showing its usefulness up to the present days in modern orthodontics.

\section{KEY WORDS}

Frankfurt plane; Natural head position; True horizontal; Cephalometry; Photogrammetry.

Int. J. Inter. Dent Vol. 14(3); 218-221, 2021.
\end{abstract}

\section{INTRODUCCIÓN}

En Odontología, la antropometría Indirecta incluye el estudio de las medidas y distancias faciales por medio de la fotogrametría y cefalometría, entre otros métodos. La fotogrametría corresponde al análisis de las distancias antropométricas en una fotografía facial, mientras que la cefalometría es el estudio que se realiza en base a mediciones craneofaciales obtenidas de trazados efectuados sobre telerradiografías laterales de cráneo estandarizadas ${ }^{(1)}$. En fotogrametría facial, la estandarización de la posición de la cabeza del individuo es fundamental. Existen protocolos que postulan el uso de la Posición Natural de Cabeza (PNC) como estado inicial, mientras que otros postulan al Plano de Frankfurt (PF), paralelo a la Horizontal Verdadera $(\mathrm{HV})$, como la posición inicial idónea(2). En cefalometría la posición en que se encuentra el paciente al momento de la toma radiográfica está inducida por el aparato radiográfico y el radiólogo busca la paralelización del PF con respecto a la HV, con el propósito de orientar el cráneo de una manera aproximada a la $\mathrm{PNC}^{(3)}$. En fotogrametría facial se propone utilizar la PNC por sobre el uso referencial del PF para establecer la postura en las fotografías de perfil. Esto debido a que existen estudios publicados para estandarizar la posición del paciente, los cuales demostraron que la postura necesaria para que el PF resultara paralelo a la HV es incómoda para la mayoría ${ }^{(4)}$.

Las radiografías laterales de cráneo que recibe el ortodoncista 
rutinariamente, pueden mostrar que la cabeza del paciente se encuentra inclinada hacia arriba o hacia abajo, no respetando el concepto de PNC. Esto puede generar la alteración de la inclinación de algunos planos cefalométricos, pudiendo perderse la apreciación real del perfil del paciente. Es por esto que cada vez que existen dudas respecto a la correcta ubicación de la cabeza en la telerradiografía lateral de cráneo, se tiene la posibilidad de controlar el problema empleando la fotografía de perfi( ${ }^{(5,6)}$. Muchas veces esta fotografía no es obtenida de manera estandarizada, por lo que tampoco refleja la PNC real del paciente, haciendo entrar al ortodoncista en el juego de "encajar" ambos exámenes diagnósticos, intentado correlacionar la cefalometría con la fotogrametría y cayendo muchas veces en distorsiones y manipulaciones que dan como resultado diagnósticos erróneos o no certeros. En este trabajo de investigación se realizó una evaluación, comparación y análisis de las radiografías laterales de cráneo con su correspondiente fotogrametría facial de perfil estandarizada en PNC, para así determinar el rango de variación promedio del $\mathrm{PF}$, uno de los referentes antropométricos faciales clave para el análisis cefalométrico.

\section{MATERIALES Y MÉTODOS}

\section{Diseño y población de estudio}

Este es un estudio de tipo observacional analítico en el que se incluyó a pacientes mayores de 18 años, atendidos en el Programa conducente al Título Profesional de Especialista en Ortodoncia de la Facultad de Odontología de la Universidad de Chile (FOUCH), entre junio de 2018 y enero de 2019. Del universo de 97 pacientes evaluados, se seleccionó por método de conveniencia a 52 voluntarios y se les solicitó que firmaran un consentimiento informado, aprobado por el Comité Ético Científico, la Dirección de Investigación y el Comité Institucional de Bioseguridad de la FOUCH. Los criterios de inclusión fueron: nuevos pacientes ingresados a la Clínica de Ortodoncia y Ortopedia Dento Máxilo Facial de la FOUCH, personas adultas entre 18 y 30 años y pacientes cuyas radiografías laterales de cráneo fueran tomadas en la unidad de radiología de la FOUCH. Mientras que los criterios de exclusión fueron: voluntarios con discapacidad motora y/o síndromes que impidieran mantener una postura estable que dificultara la toma fotográfica, pacientes con tratamiento de ortodoncia previo y radiografías laterales de cráneo tomadas en centros radiológicos externos a la $\mathrm{FOUCH}$. Al aplicar los criterios de exclusión la muestra se redujo a 34 voluntarios, de los cuales 26 fueron mujeres $(76 \%)$ y $8(24 \%)$ hombres.

\section{Antropometría Indirecta: fotografía facial}

El protocolo fotográfico utilizado fue el de Astudillo(7), el cual usó una cámara Réflex digital (Figura 1A) y un lente fijo de $100 \mathrm{~mm}$ de distancia focal. La cámara se colocó sobre un trípode a nivel de la cabeza del sujeto (Figura 1B), en donde el enfoque fotográfico fue a la altura del ala nasal. Para lograr una correcta inclinación de la cámara, se contó con la función de giroscopio electrónico integrada, que permitió obtener una imagen correctamente orientada en sentido horizontal y vertical (Figura 1C).

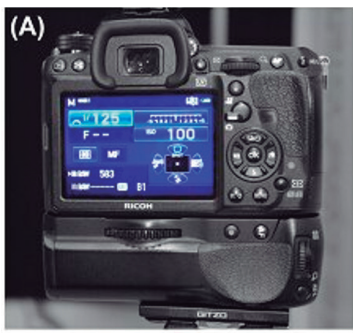

(C)
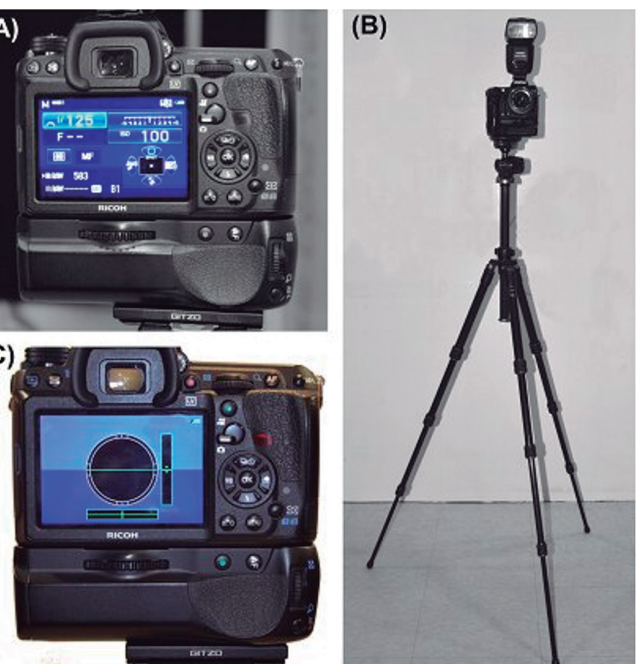

Figura 1. Características de la cámara fotográfica. (A) Cámara Réflex digital modelo K-3 con los parámetros fotográficos utilizados. (B) Cámara montada sobre un trípode modelo GT1544T Gitzo® con flash superior externo. (C) Cámara en función de giroscopio electrónico (Fuente propia 2019).

\section{Protocolo de obtención de las fotografías}

Previo a la toma fotográfica, se solicitó al voluntario el retiro de accesorios que pudieran interferir (aros, lentes, etc.) y se despejó la zona del pabellón auricular para facilitar la visualización del punto Tragion. Un único operador calibrado buscó la expresión del punto Orbitario a nive de los tejidos blandos en el lado derecho del voluntario, marcándolo con un autoadhesivo (Figura 2).

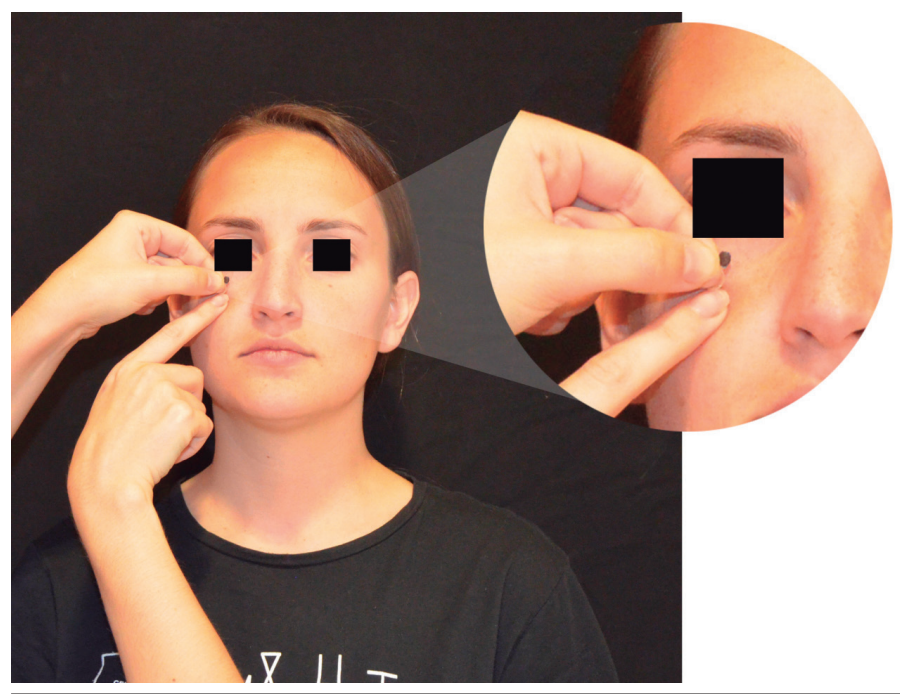

Figura 2. Identificación del punto Orbitario. Operadora marcando el punto Orbitario en una paciente. (Fuente Propia 2019).

Para alcanzar una PNC se utilizó el protocolo propuesto por Solow y Tallgren ${ }^{(8)}$. Una vez establecida, se procedió a tomar la fotografía (Figura 3).

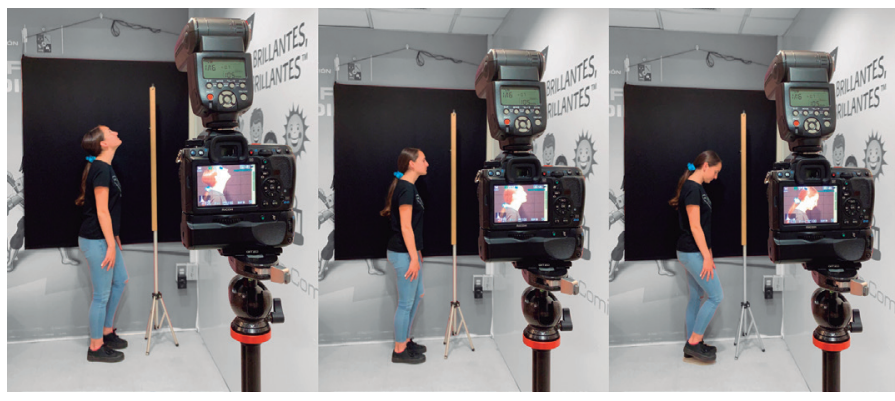

Figura 3. Posicionamiento del voluntario. Paciente ubicada de perfil según el método de autobalance propuesto por Sollow y Tallgren6, realizando movimientos de extensión y flexión de su cabeza para obtener una PNC previo a la toma fotográfica. (Fuente Propia 2019).

\section{Antropometría Indirecta: radiografía lateral de cráneo}

Se utilizaron las radiografías laterales de cráneo que forman parte de los registros para el estudio diagnóstico de los pacientes del Programa conducente al Título Profesional de Especialista en Ortodoncia y Ortopedia Dento Máxilo Facial de la FOUCH. digital

Fotogrametría y cefalometría: análisis fotográfico y radiográfico

El proceso de medición fue realizado por un único operador debidamente calibrado. Se determinaron los PF tanto en las fotografías como en las radiografías laterales de cráneo, mediante el uso del programa Adobe Photoshop CS6 ${ }^{\circledR}$. Se trazó una línea HV que pasara por el punto Tragion en las fotografías y por el punto Porion en las radiografías (Figura 4). Luego a través del software UTHSCSA ImageTool $3.0^{\circledR}$ (University of Texas Health) se determinó el ángulo formado por ambos planos (Figura 5). Se consideró a los ángulos que estuvieran sobre la HV como positivos (sentido antihorario) y bajo la HV como negativos (sentido horario). Los datos fueron tabulados en una planilla Excel (Microsoft ${ }^{\circledR}$ Excel $^{\circledR} 2010$ ) y procesados estadísticamente mediante el Software Stata $14^{\circledR} S / E^{\circledR}$. 


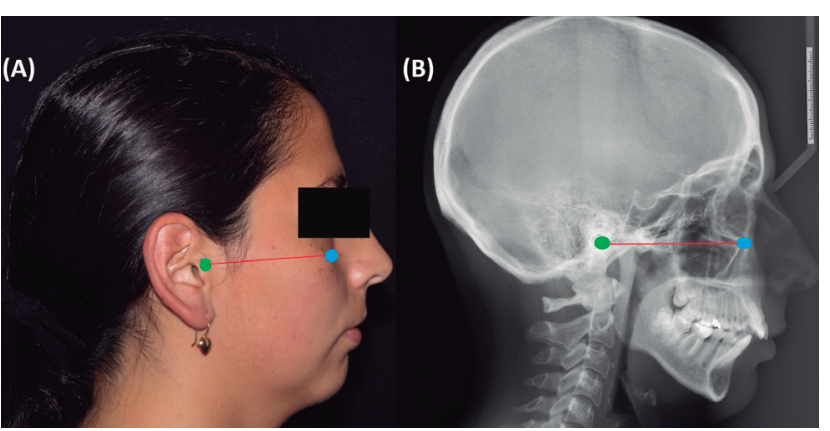

Figura 4. Determinación de planos utilizados y trazados. (A) Plano Tragion - Orbitario trazado en el paciente (Fuente Propia 2019). (B) Plano (Po-Or) trazado en la radiografía lateral de cráneo obtenida en el Departamento de Radiología FOUCH. Ambas fotografías corresponden a la misma paciente.

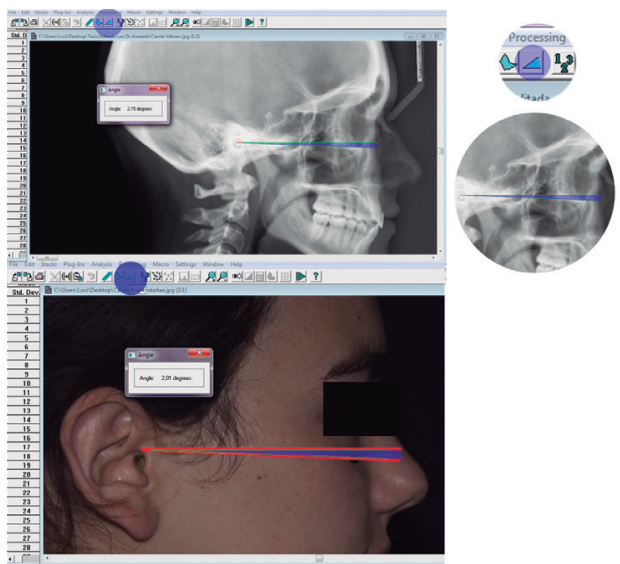

Figura 5. Obtención angular. Determinación de ángulos en fotografía y radiografía lateral de cráneo mediante el software UTHSCSA ImageTool $3.0 ®$ (University of Texas Health) en planos previamente trazados. Imagen descriptiva de uso de software utilizados, tanto fotografía como radiografía lateral de cráneo corresponden a pacientes distintos (Fuente propia 2019).

\section{RESULTADOS}

El valor promedio de la angulación del PF en PNC en la fotografía fue de $0,67^{\circ}$ (ds $0,91^{\circ}$ ) y en radiografías laterales de cráneo fue de 0 , $43^{\circ}$ (ds $\left.3,2^{\circ}\right)$. La distribución de los datos fue normal (Shapiro Wilk $p=$ $0,547)$. No hubo diferencia estadística entre ambas angulaciones (T-Test $p=0,30)$ (Tabla 1).

Tabla 1. Resumen de datos. Valores obtenidos de la angulación del PF en fotografías en PNC y radiografías laterales de cráneo estandarizadas, del total de la muestra.

\begin{tabular}{|c|c|c|c|c|c|c|}
\hline Angulación PF & $\begin{array}{l}\text { Valor } \\
\text { mínimo }\end{array}$ & $\begin{array}{l}\text { Valor } \\
\text { máximo }\end{array}$ & Promedio & $\begin{array}{l}\text { Desviación } \\
\text { estándar }\end{array}$ & $\begin{array}{l}\text { Shapiro } \\
\text { Wilk (p) }\end{array}$ & $\begin{array}{l}\text { T-Test } \\
\text { (p) }\end{array}$ \\
\hline Fotografías & $-7,17^{\circ}$ & $10,1^{\circ}$ & $0,67^{\circ}$ & $0,91^{\circ}$ & 0,547 & \multirow{2}{*}{0,30} \\
\hline $\begin{array}{l}\text { Radiografías laterales } \\
\text { de cráneo }\end{array}$ & $-6,77^{\circ}$ & $6,49^{\circ}$ & $0,43^{\circ}$ & $3,29^{\circ}$ & 0,898 & \\
\hline
\end{tabular}

\section{DISCUSIÓN}

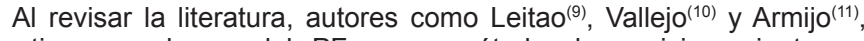
cuestionaron el uso del PF como método de posicionamiento en radiografías laterales de cráneo. Estos autores analizaron fotografías clínicas, radiografías laterales de cráneo convencionales y radiografías laterales de cráneo en PNC de distintos pacientes, con el fin de evidenciar la existencia de cambios significativos entre la postura craneofacial en PNC y la obtenida según el método de Frankfurt. Sus resultados fueron cercanos a los obtenidos en el presente estudio. En particular, destaca la coincidencia con los valores observados por Leitao ${ }^{(9)}$, quienes registraron una inclinación promedio del PF de valores menores a $1^{\circ}$.

Otras investigaciones tienden a alejarse de los resultados de este estudio, pero en su mayoría dentro de rangos que siguen siendo cercanos a la horizontal verdadera. En el estudio de Martínez ${ }^{(12)}$ registraron una diferencia de $3,75^{\circ}$ entre el PF y la horizontal, mientras que Naini(2) observó variaciones promedio de la inclinación del PF de $4,5^{\circ}$. En otra investigación, Madsen ${ }^{(13)}$ concluyó que existe una variación promedio de hasta $5^{\circ}$ entre el PF y la horizontal verdadera, rango dentro del cual se encuentran los valores obtenidos en esta investigación. Esto demuestra que la metodología aplicada en este estudio es válida, sistemática y reproducible.

Al analizar los ángulos obtenidos en las radiografías laterales de cráneo, se observó que fueron cercanos a $0^{\circ}$, donde solo 2 de 34 pacientes obtuvieron un PF paralelo a la horizontal verdadera. Tanto en fotografías como en radiografías laterales de cráneo se puede observar una mayor tendencia de angulaciones en sentido antihorario y cercanas a $0^{\circ}$, es decir, en los pacientes de esta muestra existe una predisposición a la extensión de la cabeza. Estos resultados también fueron observados por Leitao ${ }^{(9)}$ y Ferrairo ${ }^{(14)}$, quienes notaron una tendencia a la rotación de la cabeza en sentido antihorario y una disminución de la lordosis en la columna cervical cuando la cabeza se encontraba posicionada según el método de Frankfurt en el cefalostato.

Dentro de los estudios encontrados en la literatura, ninguno utilizó protocolos fotográficos estandarizados. Capon ${ }^{(15)}$ en un artículo sobre fotografía clínica y PNC, realizó una encuesta a 27 distintos profesionales del área de la salud y expertos en fotografía. Del total de los encuestados, un $77 \%$ afirmó que dirigía o "guiaba" la cabeza del paciente al tomar una fotografía. El 38,5\% utilizaba el PF como método de alineación, buscando que éste fuese paralelo al piso, ya que consideraban que era más efectivo y fácil de usar. A pesar de que el $70 \%$ se encontraba familiarizado con el concepto de PNC, solo el $12 \%$ de estos lo aplicaba en sus fotografías clínicas, siendo todos estos ortodoncistas.

Considerando lo anterior y en conjunto con los resultados obtenidos, resurge la importancia clínica del conocimiento fotográfico como examen complementario. La problemática por lo cual nace esta investigación se basa en que la cefalometría y la fotogrametría no se encuentran registradas en la misma posición de cabeza.

En un comienzo, se cuestionó el uso del PF en las radiografías laterales para orientar de manera aproximada a la PNC. Pero hoy, tras analizar los resultados de la muestra, surge una nueva interrogante: ¿es posible que el PF en PNC tenga valores cercanos a la HV y que, por ende, las manipulaciones que genera el ortodoncista para intentar correlacionar la cefalometría con la fotogrametría sean consecuencia de una fotografía clínica no estandarizada? Tras realizar el análisis y la revisión bibliográfica, se puede enfatizar que el registro fotográfico clínico debe ser estandarizado y sistematizado tanto para los parámetros fotográficos como para la obtención de una PNC, lo que evitará la tendencia del dentista de guiar la posición de la cabeza con el fin de obtener fotos más estéticas.

\section{CONCLUSIONES}

Mediante la aplicación de un protocolo sistematizado tanto para el registro fotográfico como para la obtención de la PNC, se puede lograr que el PF sea prácticamente coincidente en radiografías laterales de cráneo y en fotografías clínicas, demostrando su uso hasta el día de hoy en la ortodoncia moderna.

\section{RELEVANCIA CLÍNICA}

El plano de Frankfurt es usado comúnmente en la toma radiográfica como una referencia para posicionar la cabeza del paciente, a diferencia de la toma fotográfica en donde esta posición dependerá de la expertiz del ortodoncista. Se ha establecido una discrepancia de cinco grados en promedio entre ambas posiciones, lo que podría provocar errores en el diagnóstico clínico. Determinar la diferencia de ambos exámenes complementarios permite a los especialistas mejorar sus futuros análisis y diagnósticos cefalométricos. Los resultados de este estudio demuestran que aplicando un protocolo fotográfico estandarizado, se logra que ambas posiciones sean prácticamente coincidentes.

\section{AGRADECIMIENTOS}

Nuestros más sinceros agradecimientos al Dr. Luis Araneda Silva Docente de la Facultad de Odontología de la Universidad de Chile, por su disponibilidad y excelente disposición en su ayuda técnica al brindarme las herramientas necesarias en esta investigación para al área de la Radiología Dento Máxilo Facial.

\section{CONFLICTO DE INTERÉS}

En esta investigación ninguno de los autores presentó conflictos de interés 


\section{Bibliografía}

1. Nariño Lescay R, Alonso Becerra A, Hernández González A. Anthropometry comparative analysis of technologies for the capture of anthropometric dimensions. Rev EIA. 2016;13(26):47-59.

2. Naini FB. The Frankfort plane and head positioning in facial aesthetic analysisThe perpetuation of a myth. JAMA Facial Plast Surg. 2013;15(5):333-4. doi:10.1001/ jamafacial.2013.320

3. Proffit W, Fields H, Sarver D. Ortodoncia contemporánea. 5a ed. Elsevier; 2013.

4. Farkas LG, Bryson W, Klotz J. Is photogrammetry of the face reliable? Plast Reconstr Surg. 1980;66(3):346-55. doi:10.1097/00006534-198066030-00004

5. Lundström A, Lundström F, Lebret L, Moorrees C. Natural head position and natural head orientation: basic considerations in cephalometric analysis and research. Eur J Orthod. 1995;17(2):111-20. doi:10.1109/IEMBS.2009.5332524 6. Ozbek MM, Köklü A. Extracranial versus intracranial references in individual cephalometric analysis. Br J Orthod. 1994;21(3):259-63. doi:10.1179/bjo.21.3.259 7. Astudillo-Loyola MP, Dehghan-Manshadi-Kemm S, Vergara-Nuñez C, PeñafielEkdhal C. Son confiables las fotografías para el análisis facial en ortodoncia? Rev Clin Periodoncia Implantol Rehabil Oral. 2018;11(1):13-5. doi:10.4067/s071901072018000100013

8. Solow B, Tallgren A. Natural head position in standing subjects. Acta Odontol Scand. 1971;29(5):591-607. doi:10.3109/00016357109026337

9. Leitao P, Nanda RS. Relationship of natural head position to craniofacial morphology. Am J Orthod Dentofacial Orthop. 2000;117(4):406-17. doi:10.1016/ S0889-5406(00)70160-0

10. Vallejo J, Duque FL, Aguilar G, Ospina A, Herrera C, Arboleda LÁ. Valoración de las diferencias diagnósticas radiográficas entre la posición natural de la cabeza y la posición inducida por los auriculares del cefalostato en la angulación craneocervical. Rev Fac Odontol Univ Antioq. 2005;16:6-14. doi:10.5076

11. Armijo-Olivo S, Jara X, Castillo N, et al. A comparison of the head and cervical posture between the self-balanced position and the Frankfurt method. J Oral Rehabil. 2006;33(3):194-201. doi:10.1111/j.1365-2842.2005.01554.x

12. Ramírez DM, Jiménez JC, Ramírez EG, Paniagua HJ, Ruidíaz VC. Discrepancias en medidas cefalométricas en relación a la posición natural de la cabeza. Rev Mex Ortod. 2013;1(1):27-32. doi:10.1016/s2395-9215(16)30004-6

13. Madsen DP, Sampson WJ, Townsend GC. Craniofacial reference plane variation and natural head position. Eur J Orthod. 2008:30(5):532-40. doi:10.1093/ejo/cjn031 14. Ferrario VF, Sforza C, Germano D, Dalloca LL, Miani A. Head posture and cephalometric analyses: An integrated photographic/radiographic technique. Am J Orthod Dentofac Orthop. 1994;106(3):257-64. doi:10.1016/S0889-5406(94)70045-1 15. Capon T. Standardised anatomical alignment of the head in a clinical photography studio. A comparison between the Frankfort Horizontal and the natural head position. J Vis Commun Med. 2016;39(3-4):105-11. doi:10.1080/17453054.2 016.1246059 